Matthieu Legrand

Rick Bezemer

Asli Kandil

Cihan Demirci

Didier Payen

Can Ince

\title{
The role of renal hypoperfusion in development of renal microcirculatory dysfunction in endotoxemic rats
}

Received: 19 January 2011

Accepted: 7 May 2011

Published online: 22 June 2011

(C) The Author(s) 2011. This article is published with open access at Springerlink.com

M. Legrand and R. Bezemer contributed equally to this work.

Electronic supplementary material

The online version of this article (doi:10.1007/s00134-011-2267-4) contains supplementary material, which is available to authorized users.

M. Legrand - R. Bezemer .

A. Kandil · C. Ince

Department of Translational Physiology,

Academic Medical Center,

University of Amsterdam, Meibergdreef 9,

1105 AZ Amsterdam, The Netherlands

\section{Legrand $(\varangle) \cdot$ D. Payen}

Department of Anesthesiology and Critical

Care, Lariboisière Hospital,

Assistance Publique-Hopitaux de Paris,

University of Paris 7 Denis Diderot,

2 rue Ambroise-Paré,

75475 Paris Cedex 10, France

e-mail: matthieu.m.legrand@gmail.com

Tel.: +33-1-49958085

Fax: +33-1-49958073

\section{A. Kandil - C. Demirci}

Faculty of Science, Department of Biology, Istanbul University, 34459 Vezneciler,

Istanbul, Turkey
Abstract Purpose: To study the role of renal hypoperfusion in development of renal microcirculatory dysfunction in endotoxemic rats. Methods: Rats were randomized into four groups: a sham group $(n=6)$, a lipopolysaccharide (LPS) group $(n=6)$, a group in which LPS administration was followed by immediate fluid resuscitation which prevented the drop of renal blood flow (EARLY group) $(n=6)$, and a group in which LPS administration was followed by delayed (i.e., a 2-h delay) fluid resuscitation (LATE group) $(n=6)$. Renal blood flow was measured using a transit-time ultrasound flow probe. Microvascular perfusion and oxygenation distributions in the renal cortex were assessed using laser speckle imaging and phosphorimetry, respectively. Interleukin (IL)-6, IL-10, and tumor necrosis factor (TNF)- $\alpha$ were measured as markers of systemic inflammation. Furthermore, renal tissue samples were stained for leukocyte infiltration and inducible nitric oxide synthase (iNOS) expression in the kidney. Results: LPS infusion worsened both microvascular perfusion and oxygenation distributions. Fluid resuscitation improved perfusion histograms but not oxygenation histograms. Improvement of microvascular perfusion was more pronounced in the EARLY group compared with the LATE group. Serum cytokine levels decreased in the resuscitated groups, with no difference between the EARLY and LATE groups. However, iNOS expression and leukocyte infiltration in glomeruli were lower in the EARLY group compared with the LATE group. Conclusions: In our model, prevention of endotoxemiainduced systemic hypotension by immediate fluid resuscitation (EARLY group) did not prevent systemic inflammatory activation (IL-6, IL-10, TNF- $\alpha$ ) but did reduce renal inflammation (iNOS expression and glomerular leukocyte infiltration). However, it could not prevent reduced renal microvascular oxygenation.

Keywords Microcirculation · Blood flow · Fluid · Shock - Sepsis 


\section{Introduction}

Acute kidney injury (AKI) is a common complication in septic patients $[1,2]$. The primary resuscitation strategy for these patients is fluid resuscitation, which aims to improve organ perfusion and oxygenation and thereby prevent (multiple) organ failure. For the kidney, however, the actual contribution of hypoperfusion to the pathogenesis of AKI has recently been challenged [3, 4]. In a model of hyperdynamic sepsis in sheep [3], AKI was associated with renal vasodilation and renal hyperemia, and recovery of renal function was associated with relative vasoconstriction and decrease in renal blood flow. These findings contradict the widely accepted concept that renal hypoperfusion is the underlying cause for development of sepsis-induced AKI. Contrastingly, in animal models with lipopolysaccharide (LPS) as a key mediator of Gram-negative bacterial endotoxemia, it has been reported that LPS-induced endotoxemia is associated with renal hypoperfusion and microvascular hypoxia [6]. In addition, the hypotensive conditions associated with endotoxemia were also suggested to participate in activation of the systemic inflammatory response and consequent depression of renal perfusion and oxygenation $[7,8]$. In this line, it is possible that even a brief period of renal hypoperfusion prior to the start of fluid resuscitation could result in an ischemia-reperfusion insult that injures the renal microcirculation and potentially leads to microvascular perfusion and oxygenation heterogeneities and ultimately to AKI $[9,10]$. However, insight into renal microvascular perfusion and oxygenation heterogeneity during endotoxemia and resuscitation is still lacking, and the contribution of systemic hypotension and renal hypoperfusion during endotoxemia to renal microcirculatory dysfunction remains to be elucidated.

Endotoxemia has also been associated with impaired ability to increase oxygen extraction, even in the presence of normal or elevated cardiac output and systemic oxygen delivery $[11,12]$. This finding has led to the concept that oxygen consumption/supply dependency in sepsis is a direct result of regional rather than global oxygen delivery insufficiencies due to heterogeneity in microvascular perfusion and oxygenation [13-17]. This may explain why organ failure continues to progress even after blood pressure and cardiac output are restored $[11,12]$.

Hence, while it has been shown that fluid resuscitation decreases mortality in septic shock [5], controversy exists regarding the role of renal hypoperfusion (and thus the therapeutic value of fluid resuscitation) in sepsis-induced renal microcirculatory dysfunction. The purpose of the present study is therefore to investigate the role of renal hypoperfusion in development of renal microcirculatory dysfunction in endotoxemic rats. We hypothesized that prevention of endotoxemia-induced hypotension by immediate fluid resuscitation would prevent development of renal microcirculatory dysfunction. To this end, we studied the efficacy of immediate fluid resuscitation to prevent endotoxemia-induced systemic hypotension and renal hypoperfusion (compared with that of delayed fluid resuscitation) with respect to protection of the renal microcirculation in a rat model of LPS-induced endotoxemia. Microvascular perfusion histograms in the renal cortex were assessed using laser speckle imaging (LSI), and microvascular oxygen tension histograms were assessed using phosphorimetry. Furthermore, IL-6, IL-10, and TNF- $\alpha$ were measured as markers of systemic inflammation, and renal tissue samples were stained for leukocyte infiltration and iNOS expression in the kidney.

\section{Methods}

Animal preparation

All experiments in this study were reviewed and approved by the Animal Research Committee of the Academic Medical Center at the University of Amsterdam, The Netherlands. Care and handling of animals was performed in accordance with the guidelines of the Institutional Animal Care and Use Committees. Experiments were performed on 24 male Wistar rats (Harlan, The Netherlands) with mean \pm standard error of the mean (SEM) body weight of $351 \pm 27 \mathrm{~g}$. A more detailed description of the materials and methods is provided in the Electronic Supplementary Material (ESM). The rats were anesthetized, mechanically ventilated, and cannulated to monitor arterial blood pressure and heart rate and for continuous infusion of Ringer's lactate (Baxter, Utrecht, The Netherlands) at rate of $15 \mathrm{ml} / \mathrm{kg} / \mathrm{h}$. The left kidney was decapsulated and immobilized, and renal blood flow (RBF) and aortic blood flow (AoBF) were measured and renal vascular resistance (RVR) was calculated. The left ureter was cannulated with a polyethylene catheter for urine collection.

\section{Experimental protocol and measurements}

The rats were randomized into four groups: (1) a sham operation group (SHAM, $n=5$ ), (2) an LPS control group (LPS, $n=5$ ), (3) an LPS group with immediate fluid resuscitation (EARLY, $n=7$ ), and (4) an LPS group with delayed (i.e., $120 \mathrm{~min}$ ) fluid resuscitation (LATE, $n=7$ ). Fluid resuscitation was performed with colloid solution (Voluven ${ }^{\circledR}, 6 \%$ HES 130/0.4; Fresenius Kabi Nederland B.V., Schelle, Belgium) at rate of $20 \mathrm{ml} / \mathrm{kg} / \mathrm{h}$ in the first hour followed by $5 \mathrm{ml} / \mathrm{kg} / \mathrm{h}$ for the remainder of the experimental protocol. Arterial blood samples $(0.5 \mathrm{ml})$ were drawn at three time points: 


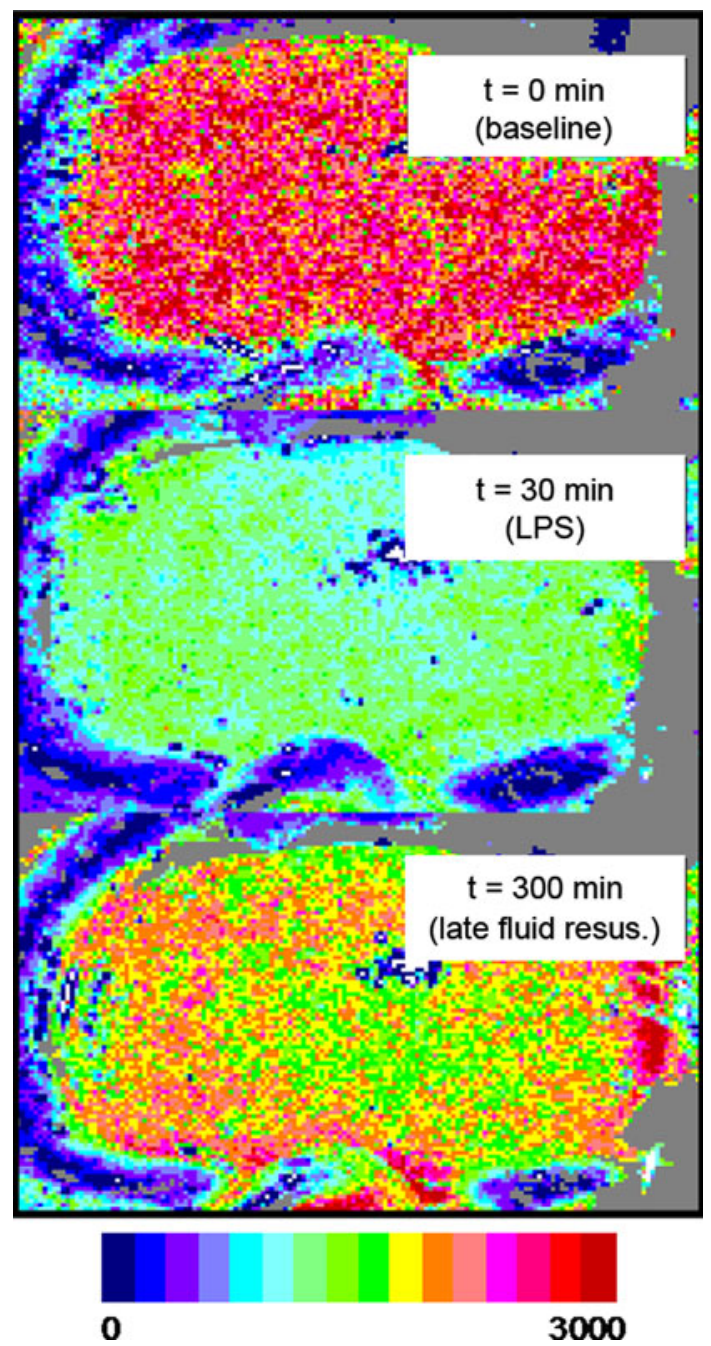

Fig. 1 Typical laser speckle imaging perfusion maps; perfusion is displayed on a 16-level color palette in a rat receiving LPS infusion $(t=30 \mathrm{~min})$ followed by delayed fluid resuscitation $(t=300 \mathrm{~min})$

(1) before infusion of LPS (baseline), (2) $120 \mathrm{~min}$ after infusion of LPS, and (3) 300 min after infusion of LPS. Microvascular perfusion and oxygenation were monitored with laser speckle imaging (LSI, Fig. 1) and phosphorimetry as described in more detail elsewhere (see [15] and [18-22], respectively). As an indicator of renal function, creatinine clearance $\left(\mathrm{Clear}_{\text {crea }}[(\mathrm{ml} / \mathrm{min})]\right)$ was assessed as an index of glomerular filtration rate. Plasma concentrations of interleukins (IL)- 6 and -10 and tumor necrosis factor (TNF)- $\alpha$ were determined using rat singleplex bead kits (Invitrogen) and read with a BioRad Bioplex 100 (BioRad). Induction of inducible nitric oxide synthase (iNOS) and infiltration of leukocytes into the kidney are considered major events in development of septic AKI. Therefore, iNOS immunostaining and anti-myeloperoxidase (MPO) staining were performed. The statistical tests for the graphs and histograms are described in the ESM.
All data are presented as mean \pm SEM; for all analyses, $p<0.05$ was considered statistically significant.

\section{Results}

All groups received $15 \mathrm{ml} / \mathrm{kg} / \mathrm{h}$ Ringer's lactate solution during the entire protocol. In addition, the EARLY group and the LATE group received $20 \mathrm{ml} / \mathrm{kg} / \mathrm{h}$ HES solution in the first hour of resuscitation, followed by $5 \mathrm{ml} / \mathrm{kg} / \mathrm{h}$ for the remaining duration of the protocol. Hence, in total, the rats in the EARLY group received $40 \mathrm{ml} / \mathrm{kg}$ in $300 \mathrm{~min}$ and the rats in the LATE group received $30 \mathrm{ml} / \mathrm{kg}$ in $300 \mathrm{~min}$.

\section{Systemic hemodynamic parameters}

The systemic hemodynamic parameters are shown in Fig. 2. Infusion of LPS induced a biphasic reduction in mean arterial pressure (MAP) in which MAP initially decreased to $\sim 65 \%$ of baseline (first $30 \mathrm{~min}$ ), then increased to approximately baseline level (next $3 \mathrm{~h}$ ), and ultimately decreased to $\sim 55 \%$ of baseline at the end of the protocol (LPS group and LATE group). In the EARLY group, fluid resuscitation prevented the initial decrease in MAP in the first phase, but in the second phase, MAP decreased to $\sim 80 \%$ of baseline. Delayed fluid resuscitation in the LATE group improved MAP from $\sim 55 \%$ (LPS group) to $\sim 70 \%$ of baseline at the end of the protocol.

Infusion of LPS induced an immediate reduction in aortic blood flow (AoBF) to $\sim 40 \%$ of baseline in the LPS group and the LATE group. In the EARLY group, fluid resuscitation resulted in $\sim 30 \%$ elevated AoBF. Delayed fluid resuscitation in the LATE group first increased AoBF to above baseline, after which it decreased to approximately baseline level. Arterial blood gas values are reported in the table in the ESM.

\section{Renal blood flow}

Renal vascular resistance (RVR) increased rapidly in the LPS and the LATE groups, while it remained stable in the SHAM and the EARLY groups. Late resuscitation was able to restore RVR to its baseline level. RBF followed an inverted pattern and decreased sharply after infusion of LPS to reach $\sim 20 \%$ of baseline at the end of the protocol. The fall in RBF was prevented in the EARLY group, in which RBF first increased above its baseline level and then slightly decreased to $\sim 85 \%$ of baseline at the end of the protocol. In the LATE group, fluid resuscitation temporarily restored RBF to its baseline level, after which it decreased to $\sim 70 \%$ of baseline. 


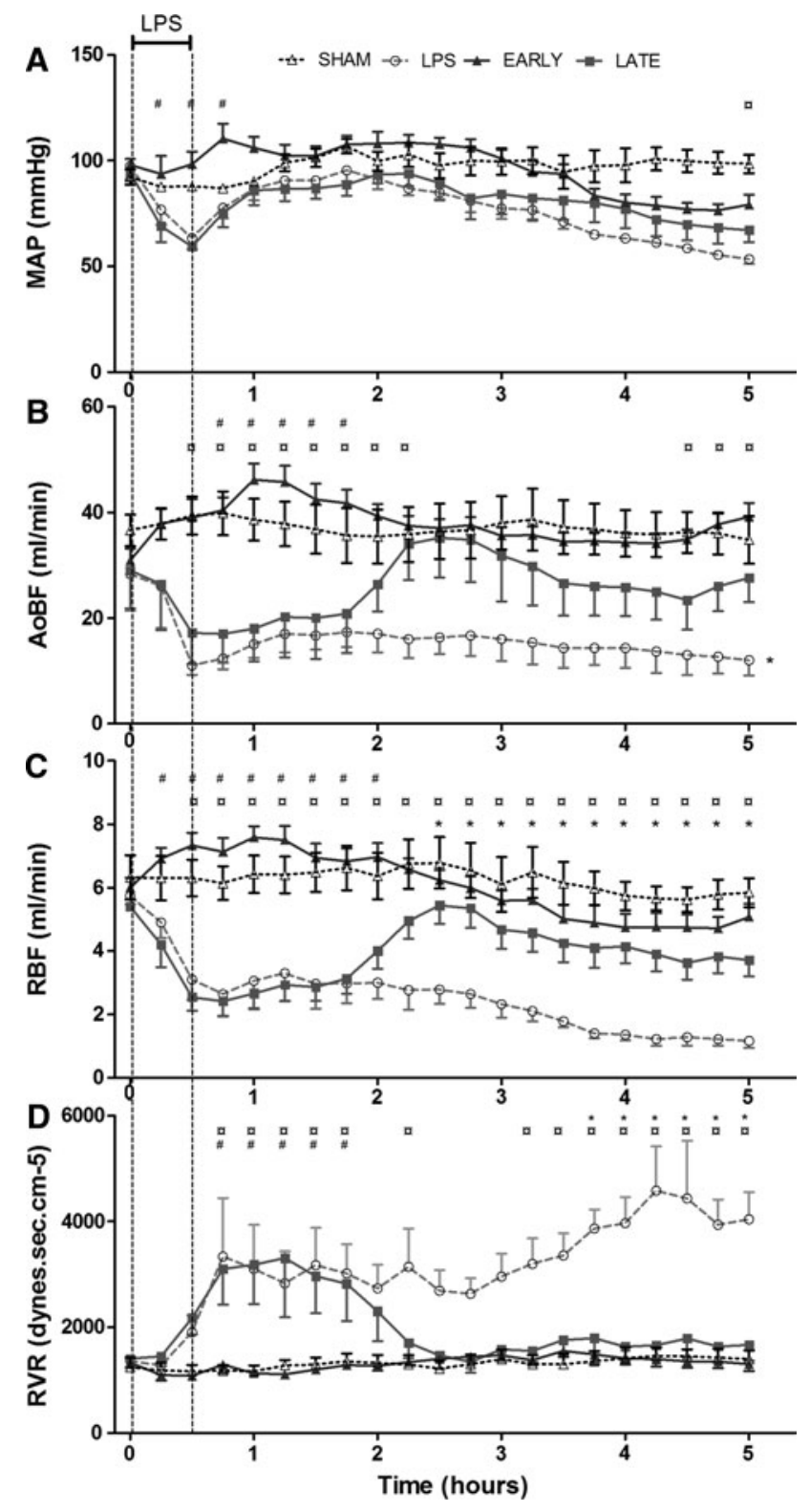

Fig. 2 Mean arterial pressure (MAP, a), aortic blood flow (AoBF, b), renal blood flow (RBF, c), and renal vascular resistance (RVR, d) during LPS infusion ( $t=0-30 \mathrm{~min}$ ) and fluid resuscitation. The LPS group received no fluid resuscitation. In the EARLY group, fluid resuscitation was started simultaneously with LPS infusion. In the LATE group, fluid resuscitation was started with a 2-h delay. ${ }^{*} p<0.05$, LPS versus LATE; ${ }^{*} p<0.05$, LATE versus EARLY; $p<0.05$, LPS versus EARLY

Renal microcirculatory parameters

\section{Renal microvascular perfusion}

LPS infusion induced a left shift in the perfusion histogram in the LPS group $(D=0.7588, p<0.05)$ and the LATE group $(D=0.7682, p<0.05)$. Immediate fluid resuscitation prevented this left shift $(D=0.0929$, $p>0.05)$. At the end of the protocol, the perfusion histograms in the EARLY group $(D=0.6920, p<0.05)$ and the LATE group $(D=0.4720, p<0.05)$ were shifted right with respect to the histogram in the LPS group. Additionally, the perfusion histograms were shifted right significantly more in the EARLY group than in the LATE group $(D=0.4444, p<0.05)$ (Fig. 3 ).

\section{Renal microvascular oxygenation}

In contrast to its effect on microvascular perfusion histograms, LPS infusion did not immediately affect the microvascular $\mathrm{PO}_{2}$ histograms in the LPS group $(D=$ $0.1918, p>0.05)$, the EARLY group $(D=0.1908$, $p>0.05)$ or the LATE group $(D=0.1798, p>0.05)$. At the end of the protocol, however, the $\mathrm{PO}_{2}$ histograms were all shifted left with respect to baseline $(D=0.4461$ and $p<0.05$ in the LPS group, $D=0.3898$ and $p<0.05$ in the EARLY group, and $D=0.2764$ and $p<0.05$ in the LATE group). No significant differences in oxygenation histograms were found at the end of the protocol between the LPS group, the EARLY group or the LATE group $(D<0.1438, p>0.05)$ (Fig. 4).

\section{Renal function}

At the end of the protocol, urine output was $1.1 \pm 0.01$, $0 \pm 0,2.0 \pm 0.5$, and $2.4 \pm 0.4 \mathrm{ml} / \mathrm{h}$ in the SHAM, LPS, EARLY, and LATE groups, respectively. While all rats in the LPS group suffered from AKI as reflected by anuria, fluid resuscitation only partially prevented AKI. Creatinine clearance rates in the EARLY and LATE groups did not differ but were statistically lower compared with the rates in the SHAM group (respectively, $0.96 \pm 0.10$ and $0.72 \pm$ $0.09 \mathrm{ml} / \mathrm{min}$ versus $1.93 \pm 0.47 \mathrm{ml} / \mathrm{min}, p<0.01$ ).

\section{Systemic inflammation}

Infusion of LPS induced marked increase in IL-6, IL-10, and TNF- $\alpha$ plasma levels (see Fig. 5 in ESM). Fluid resuscitation partially prevented the increase in IL-6, IL-10, and TNF- $\alpha$ plasma levels. The efficacy of fluid resuscitation in reducing plasma levels of these proinflammatory cytokines, however, was not dependent on its timing (i.e., no statistically significant differences between the EARLY group and the LATE group).

\section{Renal inflammation}

LPS infusion induced leukocyte infiltration in both peritubular and glomerular areas (Fig. 6 in ESM) and iNOS expression (Fig. 7 in ESM) as shown by MPO and iNOS 

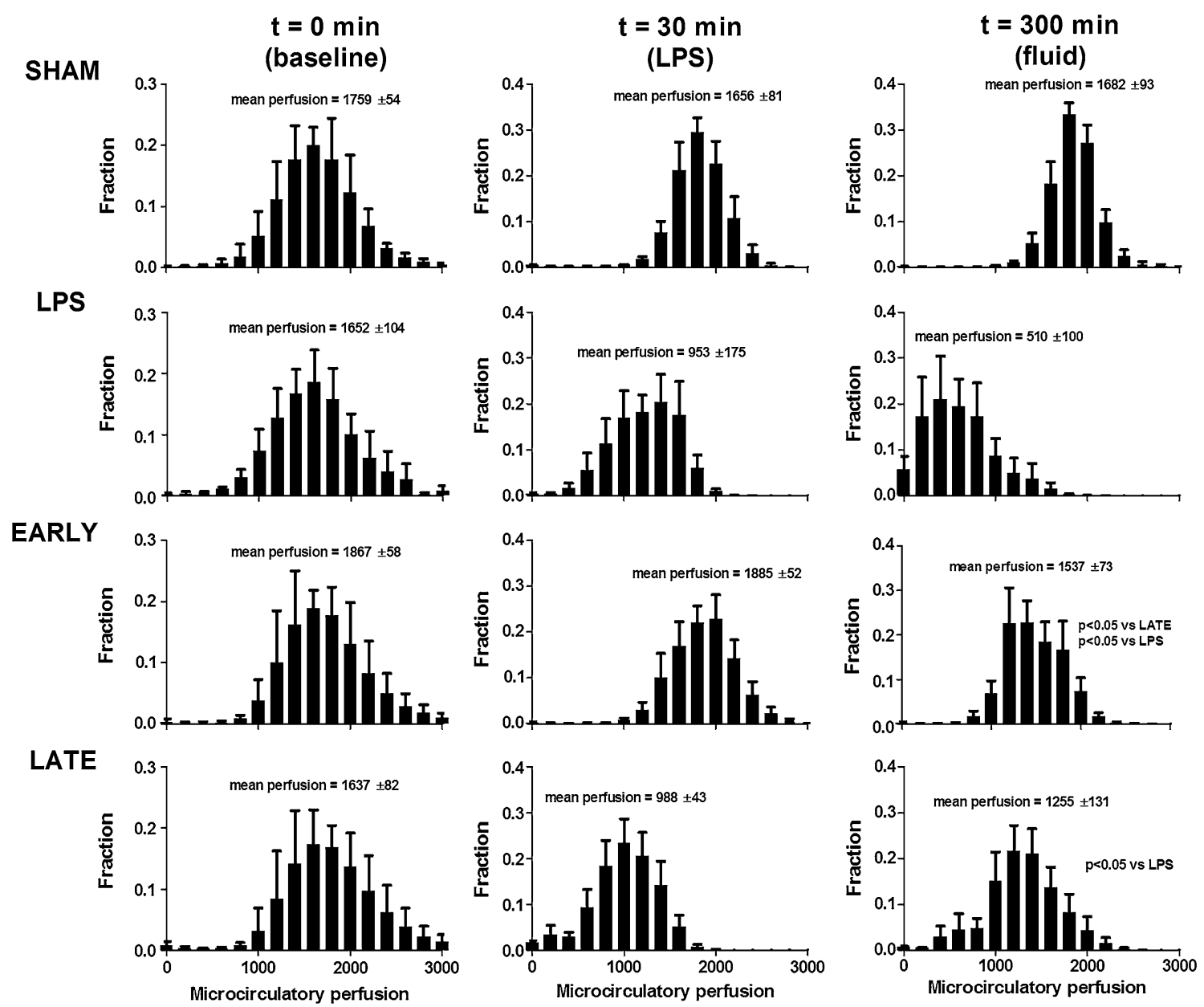

Fig. 3 Microvascular perfusion histograms in the renal cortex as measured using laser speckle imaging before (baseline) and after 30 -min infusion of LPS ( $t=30 \mathrm{~min})$ and after fluid resuscitation $(t=300 \mathrm{~min})$. The LPS group received no fluid resuscitation. In the EARLY group, fluid resuscitation was started simultaneously with LPS infusion. In the LATE group, fluid resuscitation was

staining, respectively. While delayed fluid resuscitation was not effective in reducing leukocyte infiltration and iNOS expression, these parameters were significantly improved by preventing endotoxemia-induced hypotension and renal hypoperfusion with immediate fluid resuscitation.

\section{Discussion}

The purpose of the present study is to investigate the role of renal hypoperfusion in development of renal microcirculatory dysfunction in endotoxemic rats and to test the hypothesis that prevention of endotoxemiainduced hypotension by immediate fluid resuscitation would prevent development of renal microcirculatory started with a 2-h delay. At the end of the protocol the perfusion histograms in the EARLY group and the LATE group were shifted right with respect to the histogram in the LPS group. Additionally, the perfusion histograms were shifted right significantly more in the EARLY group than in the LATE group

dysfunction. To this end, we developed an animal model in which the initial endotoxemia-induced reduction in renal blood flow was prevented by applying an immediate fluid resuscitation regimen in an LPS-induced rat model of endotoxemic shock. We have shown that prevention of renal macrovascular hypoperfusion could not fully prevent renal microcirculatory dysfunction. This indicates that endotoxemia-induced renal microcirculatory dysfunction can exist under conditions of maintained macrovascular perfusion. We have furthermore shown that prevention of hypotension by immediate fluid resuscitation could reduce renal inflammatory activation, but not systemic inflammatory activation, suggesting that endotoxemia-induced hypotension prior to fluid resuscitation leads to an ischemiareperfusion insult that potentially leads to activation of renal inflammation. 


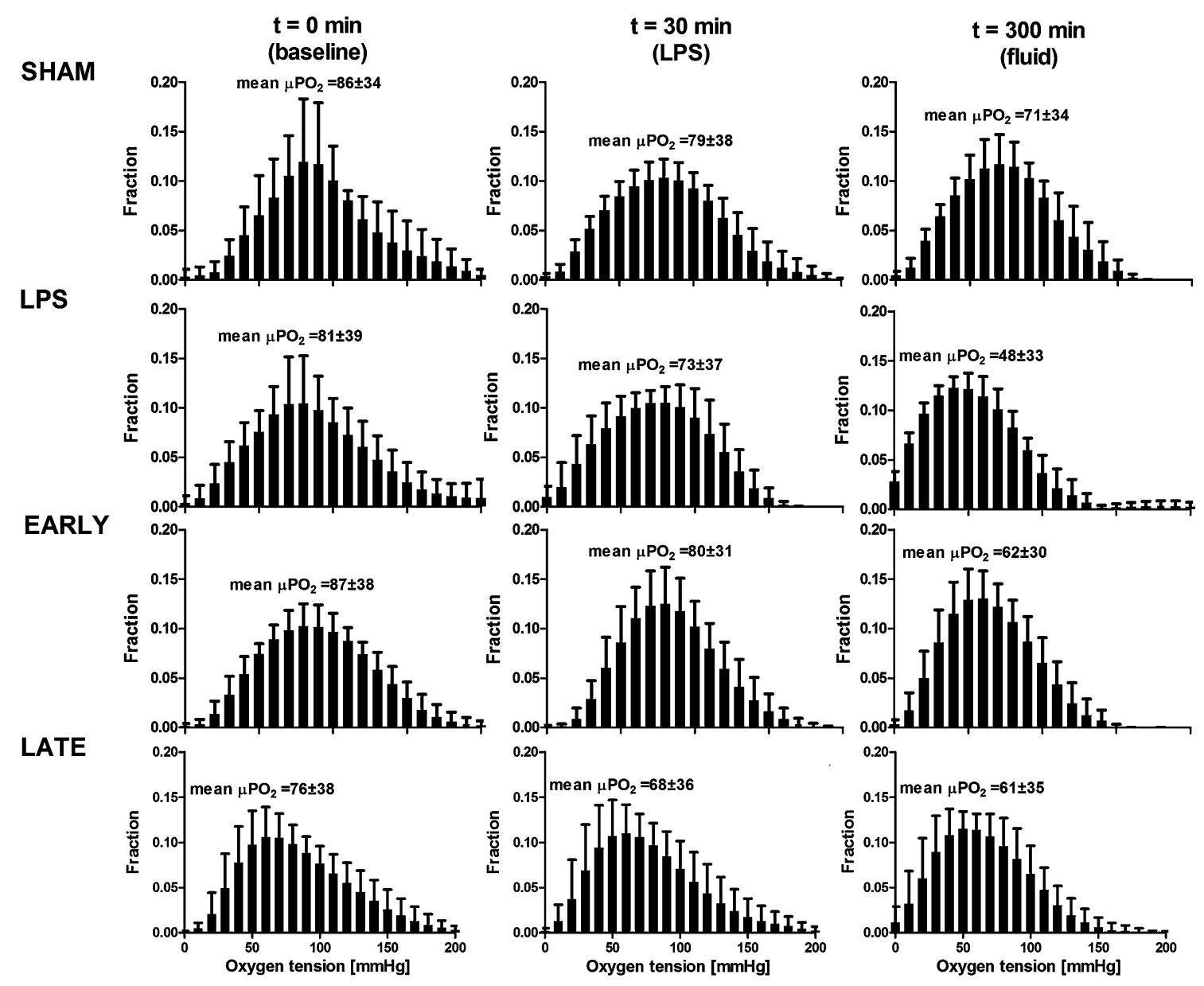

Fig. 4 Microvascular oxygen tension $\left(\mu \mathrm{PO}_{2}\right)$ histograms in the renal cortex as measured using phosphorimetry before (baseline) and after 30-min infusion of LPS $(t=30 \mathrm{~min})$ and after fluid resuscitation $(t=300 \mathrm{~min})$. The LPS group received no fluid resuscitation. In the EARLY group, fluid resuscitation was started simultaneously with LPS infusion. In the LATE group, fluid resuscitation was started with a 2-h delay. At the end of the protocol, the $\mathrm{PO}_{2}$ histograms were all shifted left with respect to baseline in the LPS, LATE, and EARLY groups. No significant differences in perfusion histograms were found at the end of the protocol between the LPS, EARLY, and LATE group
The role of renal hypoperfusion in development of AKI in septic patients remains a matter of debate. The question of whether to give these patients large fluid volume is of paramount importance given the associated increase risk of mortality in patients with AKI and positive fluid balance [23, 24]. Langenberg et al. have previously reported that AKI could develop despite an increase in renal blood flow in resuscitated septic sheep [3]. The same group has recently shown that temporary renal hypoperfusion with reduction in renal blood flow to $80 \%$ of baseline for $2 \mathrm{~h}$ did not result in alterations in renal function or histological features of renal injury [4]. Johannes et al. found that severe mechanical reduction of renal blood flow did not lead to renal microcirculatory hypoxia. However, they also discovered that LPS-induced reduction in renal blood flow led to hypoxic microcirculatory areas in the renal cortex [20]. Along these lines, the microcirculation of the renal cortex has been shown to be the severely injured in animal models of sepsis [25-30]. However, none of these studies was designed to isolate those effects of LPS directly related to hemodynamic alterations from those related to direct inflammatory response. In the present study, in contrast, we could show that endotoxemia-induced renal microcirculatory dysfunction can exist while macrovascular perfusion is maintained.

Other animal studies have shown that severe sepsis is characterized by reduction in functional capillary density and increase in blood flow heterogeneity. Goldman et al. showed that increased heterogeneity in skeletal muscle microcirculation could lead to mismatch between local oxygen supply and demand, which resulted in increased dependency of tissue oxygen utilization on the microcirculatory oxygen supply [17]. Therefore, increasing systemic oxygen delivery to normal or supernormal levels may not improve tissue oxygenation if the 
microcirculatory perfusion and oxygen supply are not properly distributed in the tissue $[8,16,17,31,32]$. Whether this phenomenon of microcirculatory perfusion heterogeneity in sepsis could apply to the kidney has not been considered previously.

We assessed microvascular perfusion distributions in the renal cortex using LSI and measured cortical microcirculatory oxygenation distributions using phosphorimetry. The advantage of LSI is its ability to provide online mapping of cortical microcirculatory perfusion with high spatial and temporal resolution and to measure perfusion distribution/ redistribution in time [15]. Using these techniques, we found that immediate fluid resuscitation, which was designed to prevent the endotoxemia-induced drop in renal blood flow, was not able to completely prevent occurrence of renal microcirculatory hypoperfusion and hypoxia. However, by means of histogram analysis, we identified that fluid resuscitation did reduce the extent of hypoxia as reflected by the reduced fraction of the first histogram bins.

There is no doubt that fluid resuscitation should be applied as early as possible to prevent AKI. However, our results suggest that completely avoiding renal macrohemodynamic failure does not prevent development of microcirculatory failure. Hence, macrohemodynamic increase in renal blood flow should be differentiated from recruitment of downstream microcirculation. Sepsisinduced microcirculatory dysfunction has been linked to several factors, including vasoconstrictor and vasodilator mediator imbalances, red blood cell and platelet aggregation, and activation of local inflammation [9, 33, 34]. Hence, sepsis affects several aspects required for proper microvascular function, thereby resulting in significant disturbances in capillary perfusion. Indeed, the results of our study show that renal microcirculatory hypoperfusion and renal hypoxia occur during endotoxemia. In addition, this microcirculatory failure appears to be relatively independent from systemic and renal macrohemodynamics, but arises from intrarenal mechanisms that may be associated with heterogeneity in the microcirculation.

A mechanism potentially responsible for the disassociation between macro- and microcirculatory parameters could be the inadequate resolution of inflammatory activation by just improving systemic hemodynamics. Despite the prevention of hypoperfusion, immediate fluid resuscitation was unable to prevent systemic inflammation. This is possibly combined with a suboptimal fluid composition leading to a disturbed plasma ion levels (e.g., hyperchloremia) and reduced oxygen-carrying capacity. Hence, the hypoperfusion and subsequent reperfusion in the early phase of endotoxemia probably contributes to the pathogenesis of endotoxemia-related AKI by forming an additional hit besides the endotoxemia-induced inflammation.

In the present study, systemic and renal inflammation responded differently to the applied fluid administration protocols. Inflammation in the renal cortex appeared to be partially prevented by immediate fluid resuscitation, as evidenced by both a decrease in leukocyte infiltration and a decrease in iNOS expression in the EARLY group compared with the LATE group. However, neither immediate nor delayed fluid resuscitation was able to prevent activation of the systemic inflammatory response, as indicated by similar increases in plasma cytokine levels (IL-6, IL-10, and TNF- $\alpha$ ). The present study, however, does not allow differentiation between those effects directly related to microcirculatory perfusion and oxygenation deficits and those related to inflammatory response, as immediate fluid resuscitation did not translate into an improvement in creatinine clearance in this short study protocol.

We are aware that our study suffers from several limitations inherent to the use of an animal model of endotoxemia. First, endotoxemic models may not reflect all situations encountered in human sepsis and may lack relevance in Gram-positive sepsis. However, it is a calibrated and reproducible model of acute inflammation that involves similar pathways and thus allows us to study the roles of renal hypoperfusion and microcirculatory dysfunction in the pathogenesis of endotoxemia-induced AKI. In this model, flow histograms slightly narrowed and oxygenation histograms slightly shifted left in time, as seen in the SHAM group. This possibly adds to the changes associated by LPS and could contribute to the lack of full recovery of the flow and oxygenation histograms. However, we could still clearly observe the effects of LPS and the differences between immediate and delayed fluid resuscitation. Thus, although the model may be slightly unstable over time, it still allows delineation between those effects of LPS directly related to hemodynamic alterations and those related to direct inflammatory response as reflected by the differences between the EARLY and the LATE group. Extrapolation of this model to clinical scenarios in terms of treatment strategies should be made with utmost caution. Instead, our study should be regarded as adding to understanding of the factors contributing to renal microcirculatory failure. Furthermore, fluid resuscitation here was very early in comparison with resuscitation in clinical scenarios. Another point of concern is that the immediate fluid resuscitation regimen might have led to altered LPS disposition in the kidney compared with the delayed resuscitation regimen. However, the applied resuscitation protocols were required to appropriately address the question of whether preventing endotoxemia-induced renal hypoperfusion would prevent renal microcirculatory failure. Although fluid resuscitation restored urine output in both resuscitated groups, creatinine clearance remained below baseline level, and whether creatinine clearance would improve in time in either of the resuscitation groups remains unidentified due to the short duration of the study protocol. Therefore, the present study only allows assessment of the hemodynamic- and inflammation-related effects of LPS in this short-term rat 
model of acute endotoxemia. Second, hydroxyl ethyl starches (HES)-based resuscitation strategies are controversial [35], and an effect of the fluid on kidney injury could not be excluded in the present study. However, only the old generation of high-molecular-weight HES molecules has been reported to be associated with acute renal failure in a dose-dependent fashion. There is no evidence for such an association with the 130/0.4 HES we used in this study. In contrast, low-molecular-weight HES molecules have been shown to have protective effects on microcirculation [36, 37]. However, in the present study, fluid resuscitation with HES-based solution led to reduction of renal microcirculatory failure and inflammation compared with nonresuscitated group. Third, changes in creatinine clearance as an index of glomerular filtration should only be regarded as a gross indicator of renal function in sepsis, which is an accepted indicator of renal function as described by the AKIN and RIFLE criteria. As more sensitive markers would be desirable, novel biomarkers are currently under investigation (e.g., neutrophil gelatinase-associated lipocalin and fatty acid binding protein), but the significance of their detection in the context of endotoxemia is a matter of concern [38,39]. Fourth, without additional data on mitochondrial function or cell viability it is unclear whether the observed alterations in the renal microcirculation were a primary or secondary cause of renal failure.

\section{Conclusions}

The present study shows that LPS-induced endotoxemia can induce alterations in microvascular perfusion and oxygenation in the renal cortex in rats, which appear to be weakly dependent on systemic and renal macrohemodynamics. Prevention of endotoxemia-induced hypotension by immediate fluid resuscitation did not prevent systemic inflammation activation (as indicated by IL-6, IL-10, and TNF- $\alpha$ plasma levels) but did reduce renal inflammation (as indicated by iNOS expression and glomerular leukocyte infiltration). However, this did not result in improved renal microcirculatory function. This renal microcirculatory failure may partially explain why renal failure progresses even after (immediate) correction of endotoxemic hypotension.

Acknowledgment This study was partially supported by an EgideLavoisier grant to Matthieu Legrand from the French Ministry of Foreign Affairs and a grant from the Nierstichting Nederland (grant number C092.2290).

Open Access This article is distributed under the terms of the Creative Commons Attribution Noncommercial License which permits any noncommercial use, distribution, and reproduction in any medium, provided the original author(s) and source are credited.

\section{References}

1. Bagshaw SM, George C, Bellomo R (2007) Changes in the incidence and outcome for early acute kidney injury in a cohort of Australian intensive care units. Crit Care 11:R68

2. Bagshaw SM, Bennett M, Haase M, Haase-Fielitz A, Egi M, Morimatsu H, D'amico G, Goldsmith D, Devarajan P, Bellomo R (2010) Plasma and urine neutrophil gelatinase-associated lipocalin in septic versus non-septic acute kidney injury in critical illness. Intensive Care Med 36:452-461

3. Bezemer R, Legrand M, Klijn E, Heger M, Post IC, van Gulik TM, Payen D, Ince C (2010) Real-time assessment of renal cortical microvascular perfusion heterogeneities using near-infrared laser speckle imaging. Opt Exp 18:15054-15061

4. Bezemer R, Faber DJ, Almac E, Kalkman J, Legrand M, Heger M, Ince C (2010) Evaluation of multiexponential curve fitting analysis of oxygen-quenched phosphorescence decay traces for recovering microvascular oxygen tension histograms. Med Biol Eng Comput 48:1233-1242
5. Bouchard J, Soroko SB, Chertow GM, Himmelfarb J, Ikizler TA, Paganini EP, Mehta RL, Program to Improve Care in Acute Renal Disease (2009) Fluid accumulation, survival and recovery of kidney function in critically ill patients with acute kidney injury. Kidney Int 76:422-427

6. Boussekey N, Darmon R, Langlois J, Alfandari S, Devos P, Meybeck A, Chiche A, Georges H, Leroy O (2010) Resuscitation with low volume hydroxyethylstarch $130 \mathrm{kDa} / 0.4$ is not associated with acute kidney injury. Crit Care 14:R40

7. Bredle DL, Samsel RW, Schumacker PT, Cain SM (1989) Critical O2 delivery to skeletal muscle at high and low PO2 in endotoxemic dogs. J Appl Physiol 66:2553-2558

8. Cabrales P, Intaglietta M, Tsai AG (2005) Increase plasma viscosity sustains microcirculation after resuscitation from hemorrhagic shock and continuous bleeding. Shock 23:549-555
9. Chvojka J, Sykora R, Krouzecky A, Radej J, Varnerova V, Karvunidis T, Hes O, Novak I, Radermacher P, Matejovic M (2008) Renal haemodynamic, microcirculatory, metabolic and histopathological responses to peritonitis-induced septic shock in pigs. Crit Care 12:R164

10. Di Giantomasso D, Morimatsu H, May CN, Bellomo R (2003) Intrarenal blood flow distribution in hyperdynamic septic shock: effect of norepinephrine. Crit Care Med 31:2509-2513

11. Dubin A, Pozo MO, Casabella CA, Palizas F Jr, Murias G, Moseinco MC, Kanoore Edul VS, Palizas F, Estenssoro E, Ince C (2009) Increasing arterial blood pressure with norepinephrine does not improve microcirculatory blood flow: a prospective study. Crit Care 13:R92

12. Dyson A, Bezemer R, Legrand M, Balestra G, Singer M, Ince C (2011) Microvascular and interstitial oxygen tension in the renal cortex and medulla studied in a 4-h rat model of lpsinduced endotoxemia. Shock (in press) 
13. Gattinoni L, Brazzi L, Pelosi P, Latini R, Tognoni G, Pesenti A, Fumagalli R (1995) A trial of goal-oriented hemodynamic therapy in critically ill patients. SvO2 Collaborative Group. N Engl J Med 333:1025-1032

14. Goldman D, Bateman RM, Ellis CG (2004) Effect of sepsis on skeletal muscle oxygen consumption and tissue oxygenation: interpreting capillary oxygen transport data using a mathematical model. Am J Physiol Heart Circ Physiol 287:H2535-H2544

15. Hoffmann JN, Vollmar B, Laschke MW, Inthorn D, Schildberg FW, Menger MD (2002) Hydroxyethyl starch $(130 \mathrm{kDa})$, but not crystalloid volume support, improves microcirculation during normotensive endotoxemia. Anesthesiology 97:460-470

16. Huang YC (2005) Monitoring oxygen delivery in the critically ill. Chest 128:554S-560S

17. Humer MF, Phang PT, Friesen BP, Allard MF, Goddard CM, Walley KR (1996) Heterogeneity of gut capillary transit times and impaired gut oxygen extraction in endotoxemic pigs. J Appl Physiol 81:895-904

18. Inan N, Iltar S, Surer H, Yilmaz G, Alemdaroglu KB, Yazar MA, Basar H (2009) Effect of hydroxyethyl starch $130 / 0.4$ on ischaemia/reperfusion in rabbit skeletal muscle. Eur J Anaesthesiol 26:160-165

19. Ince C, Sinaasappel M (1999) Microcirculatory oxygenation and shunting in sepsis and shock. Crit Care Med 27:1369-1377

20. Jang HR, Ko GJ, Wasowska BA, Rabb $\mathrm{H}$ (2009) The interaction between ischemia-reperfusion and immune responses in the kidney. $\mathrm{J}$ Mol Med 87:859-864

21. Jhanji S, Stirling S, Patel N, Hinds CJ, Pearse RM (2009) The effect of increasing doses of norepinephrine on tissue oxygenation and microvascular flow in patients with septic shock. Crit Care Med 37:1961-1966
22. Johannes T, Mik EG, Klingel K, Dieterich HJ, Unertl KE, Ince C (2008) Low-dose dexamethasone supplemented fluid resuscitation reverses endotoxin-induced acute renal failure and prevents cortical microvascular hypoxia. Shock 31:521-528

23. Johannes T, Mik EG, Ince C (2009) Nonresuscitated endotoxemia induces microcirculatory hypoxic areas in the renal cortex in the rat. Shock 31:97-103

24. Kinsey GR, Li L, Okusa MD (2008) Inflammation in acute kidney injury. Nephron Exp Nephrol 109:e102-e107

25. Langenberg C, Bellomo R, May C, Wan L, Egi M, Morgera S (2005) Renal blood flow in sepsis. Crit Care 9:R363R374

26. Langenberg C, Wan L, Egi M, May CN, Bellomo R (2007) Renal blood flow and function during recovery from experimental septic acute kidney injury. Intensive Care Med 33:1614-1618

27. Le Dorze M, Legrand M, Payen D, Ince C (2009) The role of the microcirculation in acute kidney injury. Curr Opin Crit Care 15:503-508

28. Legrand M, Almac E, Mik EG, Johannes T, Kandil A, Bezemer R, Payen D, Ince C (2009) L-NIL prevents renal microvascular hypoxia and increase of renal oxygen consumption after ischemia-reperfusion in rats. Am J Physiol Renal Physiol 296:F1109_ F1117

29. Lo LW, Vinogradov SA, Koch CJ, Wilson DF (1997) A new, water soluble, phosphor for oxygen measurements in vivo. Adv Exp Med Biol 428:651-656

30. Mårtensson J, Bell M, Oldner A, Xu S, Venge P, Martling CR (2010) Neutrophil gelatinase-associated lipocalin in adult septic patients with and without acute kidney injury. Intensive Care Med 36:1333-1340

31. Nelson DP, Samsel RW, Wood LD, Schumacker PT (1988) Pathological supply dependence of systemic and intestinal $\mathrm{O} 2$ uptake during endotoxemia. J Appl Physiol 64:2410-2419
32. Payen D, de Pont AC, Sakr Y, Spies C, Reinhart K, Vincent JL, Sepsis Occurrence in Acutely Ill Patients (SOAP) Investigators (2008) A positive fluid balance is associated with a worse outcome in patients with acute renal failure. Crit Care 12:R74

33. Rivers E, Nguyen B, Havstad S, Ressler J, Muzzin A, Knoblich B, Peterson E, Tomlanovich M, Early Goal-Directed Therapy Collaborative Group (2001) Early goal-directed therapy in the treatment of severe sepsis and septic shock. N Engl J Med 345:1368-1377

34. Saotome T, Ishikawa K, May CN, Birchall IE, Bellomo R (2010) The impact of experimental hypoperfusion on subsequent kidney function. Intensive Care Med 36:533-540

35. Schumacker PT, Samsel RW (1989) Analysis of oxygen delivery and uptake relationships in the Krogh tissue model. J Appl Physiol 67:1234-1244

36. Tyml K, Li F, Wilson JX (2008) Septic impairment of capillary blood flow requires nicotinamide adenine dinucleotide phosphate oxidase but not nitric oxide synthase and is rapidly reversed by ascorbate through an endothelial nitric oxide synthasedependent mechanism. Crit Care Med 36:2355-2362

37. Uchino S, Kellum JA, Bellomo R, Doig GS, Morimatsu H, Morgera S, Schetz M, Tan I, Bouman C, Macedo E, Gibney N, Tolwani A, Ronco C (2005) Acute renal failure in critically ill patients: a multinational, multicenter study. JAMA 294:813-818

38. Vinogradov SA, Grosul P, Rozhkov V, Dunphy I, Shuman L, Dugan BW, Evans S, Wilson DF (2003) Oxygen distributions in tissue measured by phosphorescence quenching. Adv Exp Med Biol 510:181-185

39. Walley KR (1996) Heterogeneity of oxygen delivery impairs oxygen extraction by peripheral tissues: theory. J Appl Physiol 81:885-894 